\title{
Latest features in GaBI Journal, 2014, Issue 3
}

\section{Professor Philip D Walson, MD}

This issue of the GaBI Journal begins with editorial comments from our Deputy Editor-in-Chief Dr Robin Thorpe concerning the Review Article by Azevedo et al. covering biosimilar regulations and their implementation in some Latin American countries.

Three Original Research papers describe real life clinical examples relating to the use and evaluation of follow-on products respectively; the first manuscript from Cohen et al. presents questionnaire data collected from two specific groups of US stakeholders: healthcare payers and key opinion leader physicians. The goals of the study were to identify attitudes towards and barriers to use that are likely to influence the use of selected biosimilars once they are approved for use in the US. The rationale used to select the specific products to ask about, to choose the qualitative Likert scale questions asked, and to select the 36 payers and 42 physicians who were sent the questionnaires were all very well justified and described. The authors also gave the actual response rate (eight payers and 14 physicians) as well as information on responder characteristics. The method and result details provided allow readers to assess how well the results can be extrapolated and allowed the work to be classified as original research.

The second research paper presents data from a retrospective chart review done by Professor Rottembourg et al. for his dialysis patients following the 'automatic' substitution by hospital pharmacy of a follow-on iron sucrose product. These data raise questions about the comparability of follow-on iron sucrose nonbiological complex drugs (NBCDs), about the role of treating physicians in decisions about the use of such follow-on products, as well as about the validity of cost saving assumptions that are being used to justify such substitutions. While the paper deals with an NBCD the questions raised are also important for both generic and biosimilar products. It is hoped that our readers will submit both comments about this report as well as their experiences with either successful or unsuccessful substitutions of follow-on products.

The third research paper by Janknegt et al. discusses the use of the SOJA (System of Objectified Judgement Analysis) method to make decisions on the selection of which GnRH agonists and antagonists to use in patients with prostate cancer as therapy. The SOJA method uses both published data and the input from multiple stakeholders, including clinicians, to make decisions about which product or products should be selected. The problems described by Rottembourg et al. would likely have been avoided had the SOJA method been used to assess iron sucrose products before their automatic substitution.

This issue also contains three manuscripts describing regional issues related to biosimilar regulation and use, a Perspective in Canada by Klein et al. from Health Canada, an Original Research in China by Zeng et al., and a Review Article by Azevedo et al. about the situation in Latin America commented on by Dr Robin Thorpe in the previously mentioned Editorial.

A summary of an Interview with representatives of Amgen and Actavis provides insights into a number of topics including their collaborative commercialization agreement and their views on the development, efficacy and safety testing, and educational components
Official Journal of the

GENERICS AND BIOSIMILARS INITIATIVE GaBi Journal



of their partnership to develop effective anti-cancer monoclonal antibodies.

Finally, another questionnaire study was selected for inclusion as a Pharma News article. This article presents results from a questionnaire survey conducted by the European Crohn's and Colitis Organisation (ECCO) at their 2013 meeting. Unfortunately, it is not possible to know how representative responses were because the authors present only the number of people who responded and not the number asked to respond. Nevertheless, the data are similar to the US study and suggest that confidence in biosimilars used to treat inflammatory bowel disease is often lacking. What is not clear is whether or how much of this lack of confidence is justified or correctable by collection of data and/or action by regulators or manufacturers but it is hoped that the data from both the US and EU should prompt careful consideration.

I again urge readers to both comment on these and any other GaBI Journal published papers as well as to contribute their own relevant material.

Professor Philip D Walson, MD Editor-in-Chief, GaBiJournal

DOI: 10.5639/gabij.2014.0303.026

Copyright @ 2014 Pro Pharma Communications International 